\title{
Focal myositis: an unusual cause of bilateral upper eyelid swellings
}

\author{
K.L. Lim, K. Robson ${ }^{1}$ and R.J. Powell \\ Departments of Immunology and ${ }^{1}$ Neurohistopathology, University Hospital, Queen's Medical Centre, \\ Nottingham, UK
}

\begin{abstract}
Summary: Focal myositis is considered a rare self-limiting inflammatory swelling of skeletal muscle. Focal involvement of individual muscle or muscle groups with severe myopathic and inflammatory changes characterizes the histology. We report the development of progressive focal myositis in both upper eyelids of a 34 year old man that responded to immunosuppressive therapy.
\end{abstract}

\section{Introduction}

Focal myositis, described by Heffner and colleagues in $1977,{ }^{1}$ is considered to be a benign, inflammatory swelling of skeletal muscle that may mimic a malignant soft tissue tumour. Fewer than 30 of these lesions have been reported, the majority of which were located in the limbs. We report the development of focal myositis in both upper eyelids of a man which responded rapidly to immunosuppressive therapy.

\section{Case report}

A 34 year old West Indian presented to Eye Casualty with a 2 month history of discomfort and progressive swelling of his left upper eyelid. A course of antibiotics was prescribed for a suspected infected cyst. However, the left eyelid swelling progressed and was biopsied 6 months after presentation. At operation, the mass appeared to arise from the musculature of the upper eyelid and two biopsies, the largest measuring $1.5 \mathrm{~cm}$ in maximum extent, were obtained. Microscopic examination demonstrated active muscle destruction, interstitial fibrosis and oedema, and lymphocytic infiltration associated with a marked lymphocytic vasculitis (Figures 1 and 2).

Without other treatment or intervention, there was further gradual increase in size of the left eyelid mass over the next 3 months and a month prior to

Correspondence: K.L. Lim, M.B., M.R.C.P., Clinical Immunology Unit, F Floor, University Hospital, Queen's Medical Centre, Nottingham NG7 2UH, UK.

Accepted: 20 May 1993

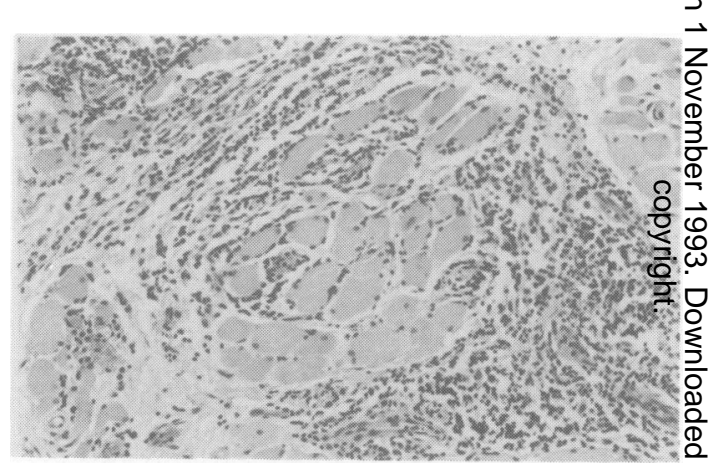

Figure 1 Eyelid biopsy showing oedema and lymphocytic infiltration $(\mathrm{H} \& \mathrm{E}, \times 240)$.

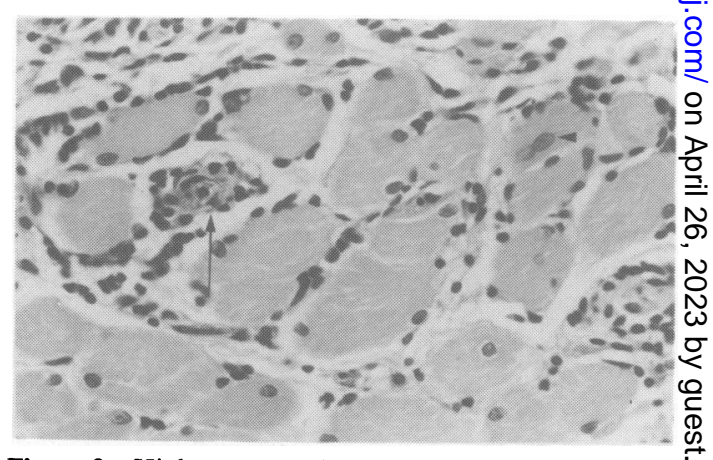

Figure 2 Higher power showing vessel cuffing (arrow) and regenerating fibre with vesicular nuclei (arrowhead) (H\&E, $\times 600)$. 
him being seen in the Clinical Immunology Unit a similar lesion developed in his right upper eyelid. There was no specific history of trauma or infection. There were no symptoms of a systemic illness and no personal or family history of soft tissue tumours, connective tissue diseases, other autoimmune diseases or neuromuscular disorders.

Physical examination showed diffuse enlargement with overlying erythema and oedema of both upper eyelids (Figure 3). The bilateral masses were tender to palpation. Clinical involvement of other orbital structures was absent and the remainder of the physical examination was normal. Full blood count, urea and electrolytes, liver function tests and thyroid function tests were within normal range. Serial erythrocyte sedimentation rate, creatine kinase, plasma complement $\mathrm{C} 3, \mathrm{C} 4$ and $\mathrm{C} 3$ degradation products and immunological tests including ANA, ENA, anti-dsDNA and ANCA were persistently normal or negative. Radiographs of the chest and sinuses were reported as normal. A computed tomographic (CT) scan of the orbits showed that the lesions were pre-septal, separate from the lacrimal glands with no involvement of the globes, optic nerves, extra ocular muscles or underlying bone. A needle muscle biopsy of the left quadriceps was normal - that is, did not show occult myositis or lymphocytic vasculitis.

The patient was commenced on prednisolone $20 \mathrm{mg}$. There was rapid resolution of his lesions, including the lesion in the right upper eyelid that had not been biopsied, within 2 weeks and the dose of prednisolone was gradually reduced and discontinued. He suffered a flare of his symptoms when he was on less than $10 \mathrm{mg}$ of prednisolone daily, requiring a transient increase in his prednisolone dosage and the addition of azathioprine $150 \mathrm{mg}$ daily. The latter drug was continued for a total of 9 months. A year later, there has been no recurrence of his lesions and he had not developed classical polymyositis or a systemic vasculitic illness.

\section{Discussion}

To our knowledge, focal myositis of the musculature of the upper eyelids has not been previously reported. In Heffner's original series of 16 patients, eight lesions were in the thigh, four in the calf, two in the thorax and one each in the abdomen and forearm. Other sites reported in the literature since then have included the abdominal wall, ${ }^{2}$ neck, ${ }^{3,4}$ tongue, ${ }^{5,6}$ perioral muscles ${ }^{7}$ and hand. ${ }^{8}$

It appears that the condition can affect any age group and has an equal sex distribution. Typically the major presenting feature is a rapidly expanding soft tissue mass which might be tender to palpation. The majority of lesions are present from 2 to 8

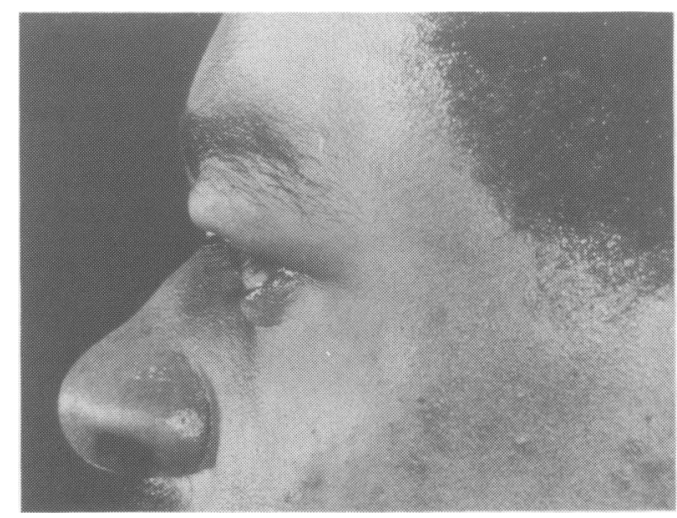

Figure 3 Lateral view of left upper eyelid swelling in patient before treatment.

weeks before a diagnosis is made. Our patient, like others reported previously, was in good general health and had normal laboratory tests including creatine kinase. Contrary to the reported benign nature of focal myositis, our patient had progressive disease with involvement of his contralateral upper eyelid 12 months after his initial symptoms. Initially there was rapid response to prednisolone therapy but longer term treatment with azathioprine was subsequently required to maintain remission of his disease. To date, steroid therapy used to induce rapid remission has only been reported in one case of focal myositis. ${ }^{9}$

Focal myositis needs to be differentiated from other soft tissue tumours involving skeletal muscles such as neoplasms, nodular faciitis, proliferative myositis and myositis ossificans. Eosinophilic myositis may be focal in presentation ${ }^{10}$ and was excluded on the basis of normal blood eosinophil counts and lymphocytic, and not eosinophilic, infiltration seen in the muscle biopsy specimen. Eyelid swelling may also be the presenting feature of orbital myositis ${ }^{11}$ but this patient never had clinical or radiographic evidence of extra ocular muscle involvement. Hence the diagnosis of focal myositis was made on the clinical history and the typical histological appearances.

The aetiology of this condition is unknown. Trauma or muscular injuries have been reported to be the initiating insult in a number of patients. However, symmetrical involvement of a paired structure observed in our patient similar to other conditions such as autoimmune Addison's disease and vitiligo may suggest a common aetiopathogenesis. 


\title{
References
}

1. Heffner, R.R., Armbrustmacher, V.W. \& Earl, K.M. Focal myositis. Cancer 1977, 40: 301-306.

2. Hansen, L.B., Baekgaard, N. \& Reske, N.E. Focal myositis of the rectus abdominal muscles. Acta Chir Scand 1985, 151: 77-80.

3. Isaacson, G., Chan, K.H. \& Heffner, R.J. Focal myositis. A new cause for the paediatric neck mass. Arch Otolaryngol Head Neck Surg 1991, 117: 103-105.

4. Shapiro, M.J., Applebaum, H. \& Besser, A.S. Cervical focal myositis in a child. J Pediatr Surg 1986, 21: 375-376.

5. Azuma, T., Komori, A. \& Nagayama, M. Focal myositis of the tongue. J Oral Maxillofac Surg 1987, 45: 953-955.

6. Takita, M.A., Kawamoto, T.O., Nogami, H., Takada, M., Onishi, S. \& Sakuda, M. Focal myositis of the tongue: report of a case. J Osaka Univ Dent Sch 1985, 25: 161-169.
7. Ellis, G.L. \& Brannon, R.B. Focal myositis of the perioral musculature. Oral Surg 1979, 48: 337-341.

8. Maguire, J.J., Milford, L.W. \& Pitcock, J.A. Focal myositis $\subsetneq$ in the hand. $J$ Hand Surg Am 1988, 13: 140-142.

9. Chiba, S., Miyano, N., Matsumoto, H., Chiba, T. \& $\stackrel{\oplus}{+}$ Wakabayashi, J. [A case of isolated focal myositis successfully treated by arterial injection of prednisolone-its findings $\bar{\sigma}$ by CT scan and MRI]. Rinsho Shinkeigaku 1990, 30: $\overline{\bar{\omega}}$ $1261-1265$.

10. Agrawal, B.L. \& Giessen, P.C. Eosinophilic myositis. An $\cong$ unusual cause of pseudotumour and eosinophilia. JAMA 1981, 246: $70-71$.

11. Li, J.T., Garrity, J.A., Kephart, G.M. \& Gleich, G.J. $\vec{\circ}$ Refractory periorbital oedema in a 29 -year-old man [clinical conference]. Ann Allergy 1992, 69: 101-105.

\section{Candida lusitaniae causing fatal meningitis}

\author{
P.S.A. Sarma ${ }^{1}$, P. Durairaj ${ }^{2}$ and A.A. Padhye ${ }^{3}$
}

Departments of ${ }^{1}$ Medicine and ${ }^{2}$ Microbiology, Jawaharlal Nehru Hospital \& Research Centre, Bhilainagar-9, Madhya Pradesh 490006, India and ${ }^{3}$ Division of Mycotic and Bacterial Diseases, Center fळ Infectious Diseases, Centers for Disease Control, Public Health Service, US Department of Health and Human Services, Atlanta, Georgia, USA

Summary: Fatal meningitis due to Candida lusitaniae in a 35 year old previously healthy man is described. $C$. lusitaniae is an opportunistic fungal pathogen reported infrequently in the English literature. This is the third case report of meningitis and the first fatal infection in an adult from Central India due to C. lusitaniae known to the authors.

\section{Introduction}

Candida lusitaniae van Uden et do Carmo-Sousa, originally isolated from the gastrointestinal tracts of warm-blooded animals, ${ }^{1}$ has recently been found to colonize rarely the gastrointestinal, respiratory and urinary tracts and skin of hospitalized patients. $^{2}$ This organism shares morphological, biochemical and other characteristics with other species of the genus Candida, such as $C$. tropicalis and $C$. parapsilosis. ${ }^{3}$ More recently, C. lusitaniae has been recovered from a variety of human clinical specimens such as respiratory and genitourinary secretions, stool, blood, pleural fluid, kidneys, lungs, bone $e^{2,45}$ and cerebrospinal fluid. ${ }^{6,7}$

Correspondence: P.S.A. Sarma, M.D., MIG II-437, Amdinagar, HUDCO, Bhilainagar-9, Madhya Pradesh 490006, India.

Accepted: 22 March 1993
The reports of a premature infant ${ }^{7}$ and a 2 year old white male ${ }^{6}$ with $C$. lusitaniae sepsis and meningitis are the only examples of meningeal 3 . infection caused by this organism, of which we are 8 aware. Blinkhorn et al. ${ }^{8}$ in their review did not $\frac{}{3}$ include any additional patient with this form of 0 C. lusitaniae disease. The present report describes a third case of meningitis and a first fatal case caused $\frac{}{0}$ by this emerging pathogen in a non-white patient from central India.

\section{Case report}

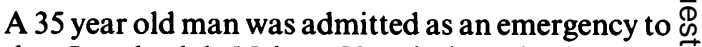
the Jawaharlal Nehru Hospital and Research Centre, Bhilainagar, Madhya Pradesh, India, with 0 a 8 day history of fever and unconsciousness of one 